\title{
ON THE AVERAGE NUMBER OF REPRESENTATIONS OF AN INTEGER AS A SUM OF LIKE PRIME POWERS
}

\author{
MARCO CANTARINI, ALESSANDRO GAMBINI, ALESSANDRO ZACCAGNINI
}

\begin{abstract}
We investigate the average number of representations of a positive integer as the sum of $k+1$ perfect $k$-th powers of primes. We extend recent results of Languasco and the last Author, which dealt with the case $k=2$ [6] and $k=3$ [5] respectively. We use the same technique to study the corresponding problem for sums of just $k$ perfect $k$-th powers of primes.
\end{abstract}

\section{INTRODUCTION}

The problem of representing a large integer $n$, satisfying suitable congruence conditions, as a sum of a prescribed number of powers of primes, say $n=p_{1}^{k_{1}}+\cdots+p_{s}^{k_{s}}$, is classical. Here $k_{1}, \ldots, k_{s}$ denote fixed positive integers. This class of problems includes both the binary and ternary Goldbach problem, and Hua's problem. If the density $\rho=k_{1}^{-1}+\cdots+k_{s}^{-1}$ is large and $s \geq 3$, it is often possible to give an asymptotic formula for the number of different representations the integer $n$ has. When the density $\rho$ is comparatively small, the individual problem is usually intractable and it is reasonable to turn to the easier task of studying the average number of representations, if possible considering only integers $n$ belonging to a short interval $[N, N+H]$, say, where $H \geq 1$ is as small as possible.

Here we deal with the average number of representations of a positive integer $n$ as the sum of $k+1$ perfect $k$-th powers of prime numbers. The case $k=2$ (actually, to be exact a slightly more general problem) has been studied in [6], while the case $k=3$ has been studied in [5]. Here we give a uniform and simpler proof which is valid for general $k \geq 2$. Let

$$
R_{k}(n)=\sum_{n=m_{1}^{k}+\cdots+m_{k+1}^{k}} \Lambda\left(m_{1}\right) \cdots \Lambda\left(m_{k+1}\right),
$$

where $\Lambda$ is the von Mangoldt function, that is, $\Lambda\left(p^{m}\right)=\log (p)$ if $p$ is a prime number and $m$ is a positive integer, and $\Lambda(n)=0$ for all other integers.

Theorem 1.1. Let $k \geq 2$ be a fixed integer. For every $\varepsilon>0$ there exists a constant $C=C(\varepsilon)>0$, independent of $k$, such that

$$
\sum_{n=N+1}^{N+H} R_{k}(n)=\Gamma\left(1+\frac{1}{k}\right)^{k} H N^{1 / k}+O_{k}\left(H N^{1 / k} \exp \left\{-C\left(\frac{\log N}{\log \log N}\right)^{1 / 3}\right\}\right)
$$

as $N \rightarrow+\infty$, uniformly for $N^{1-5 /(6 k)+\varepsilon}<H<N^{1-\varepsilon}$, where $\Gamma$ is the Euler Gamma-function.

Theorem 1.2. Let $k \geq 2$ be a fixed integer and assume that the Riemann Hypothesis (RH) holds. Then

$$
\sum_{n=N+1}^{N+H} R_{k}(n)=\Gamma\left(1+\frac{1}{k}\right)^{k} H N^{1 / k}+O_{k}\left(\Phi_{k}(N, H)\right)
$$

Date: August 29, 2019.

2010 Mathematics Subject Classification. Primary 11P32. Secondary 11P55, 11P05.

Key words and phrases. Waring-Goldbach problem; Hardy-Littlewood method. 
uniformly for $H=\infty\left(N^{1-1 / k} L^{3}\right)$ with $H=o(N)$, where $f=\infty(g)$ means $g=o(f), L=\log N$ and $\Phi_{k}(N, H)=N L^{3}+H^{2} N^{1 / k-1}+H^{1 / 2} N^{1 / 2+1 /(2 k)} L+H N^{1 /(2 k)} L^{3 / 2}$.

The density of this problem is $1+1 / k$. Theorems 1.1 and 1.2 contain as special cases the results in [6] and [5]. The limitation in Theorem 1.1 is due to the corresponding one in Lemma 3.1, whereas the limitation in Theorem 1.2 is the expected one.

The main new ingredient is the use of the elementary identity

$$
x^{k+1}-y^{k+1}=(x-y)^{2} \sum_{j=1}^{k} j x^{k-j} y^{j-1}+(k+1)(x-y) y^{k},
$$

which is valid for integral $k \geq 1$. We will show in $\$ 4$ below that this identity can be used quite effectively to circumvent the need for sharp bounds for exponential sums. In fact, we manage to obtain the "expected" limitations (in view of Lemma 3.1) for the length $H$ of the "short interval," both in the unconditional and in the conditional case.

We remark that the use of identity (2) allows us to treat also the average value of the number of representations of an integer as a sum of just $k$ perfect $k$-th powers of prime numbers, which is a problem of density 1 . Let

$$
R_{k}^{\prime}(n)=\sum_{n=m_{1}^{k}+\cdots+m_{k}^{k}} \Lambda\left(m_{1}\right) \cdots \Lambda\left(m_{k}\right)
$$

We can prove the following results, which will also appear in a forthcoming paper by A. Languasco with a proof along the lines of [5].

Theorem 1.3. Let $k \geq 2$ be a fixed integer. For every $\varepsilon>0$ there exists a constant $C=C(\varepsilon)>0$, independent of $k$, such that

$$
\sum_{n=N+1}^{N+H} R_{k}^{\prime}(n)=\Gamma\left(1+\frac{1}{k}\right)^{k} H+O_{k}\left(H \exp \left\{-C\left(\frac{\log N}{\log \log N}\right)^{1 / 3}\right\}\right)
$$

as $N \rightarrow+\infty$, uniformly for $N^{1-5 /(6 k)+\varepsilon}<H<N^{1-\varepsilon}$.

Theorem 1.4. Let $k \geq 2$ be a fixed integer and assume that the Riemann Hypothesis holds. Then

$$
\sum_{n=N+1}^{N+H} R_{k}^{\prime}(n)=\Gamma\left(1+\frac{1}{k}\right)^{k} H+O_{k}\left(\Phi_{k}^{\prime}(N, H)\right),
$$

uniformly for $H=\infty\left(N^{1-1 / k} L^{3}\right)$ with $H=o(N)$, where $\Phi_{k}^{\prime}(N, H)=N^{1-1 / k} L^{3}+H^{2} N^{-1}+$ $H^{1 / 2} N^{1 / 2-1 /(2 k)} L+H^{1-1 / k} N^{1 /(2 k)} L^{3 / 2}$.

We do not give the full detailed proofs of Theorems 1.3 and 1.4, but just a short summary in $\$ 6$. The starting point is the use of identity (2) with $k$ in place of $k+1$. The case $k=2$ of both results is proved in Languasco \& Zaccagnini [3].

\section{DEFINITIONS AND PREPARATION FOR THE PROOFS}

For real $\alpha$ we write $\mathrm{e}(\alpha)=\mathrm{e}^{2 \pi \mathrm{i} \alpha}$. We take $N$ as a large positive integer, and write $L=\log N$ for brevity. Let $z=1 / N-2 \pi \mathrm{i} \alpha$ and

$$
\widetilde{S}_{k}(\alpha)=\sum_{n \geq 1} \Lambda(n) \mathrm{e}^{-n^{k} / N} \mathrm{e}\left(n^{k} \alpha\right)=\sum_{n \geq 1} \Lambda(n) \mathrm{e}^{-n^{k} z} .
$$

Thus, recalling definition (1) and using (4), for all $n \geq 1$ we have

$$
R_{k}(n)=\sum_{n_{1}^{k}+\cdots+n_{k+1}^{k}=n} \Lambda\left(n_{1}\right) \cdots \Lambda\left(n_{k+1}\right)=\mathrm{e}^{n / N} \int_{-1 / 2}^{1 / 2} \widetilde{S}_{k}(\alpha)^{k+1} \mathrm{e}(-n \alpha) \mathrm{d} \alpha .
$$


It is clear from the above identity that we are only interested in the range $\alpha \in[-1 / 2,1 / 2]$. We record here the basic inequality

$$
|z|^{-1} \ll \min \left\{N,|\alpha|^{-1}\right\} .
$$

We also need the following exponential sum over the "short interval" $[1, H]$

$$
U(\alpha, H)=\sum_{m=1}^{H} \mathrm{e}(m \alpha)
$$

where $H \leq N$ is a large integer. We recall the simple inequality

$$
|U(\alpha, H)| \leq \min \left\{H,|\alpha|^{-1}\right\} .
$$

With these definitions in mind and recalling (5), we remark that

$$
\sum_{n=N+1}^{N+H} \mathrm{e}^{-n / N} R_{k}(n)=\int_{-1 / 2}^{1 / 2} \widetilde{S}_{k}(\alpha)^{k+1} U(-\alpha, H) \mathrm{e}(-N \alpha) \mathrm{d} \alpha,
$$

which is the starting point for our investigation. The basic strategy is to replace $\widetilde{S}_{k}(\alpha)$ by its expected main term, which is $\Gamma(1+1 / k) / z^{1 / k}$, and estimating the ensuing error term by means of identity (2). We use trivial bounds for $\widetilde{S}_{k}$ and $z^{-1 / k}$ and Lemma 3.1 to majorise each term in the sum on the right-hand side of (2), and the Cauchy-Schwarz inequality and the same Lemma again to majorise the summand on the far right. Of course, we may use Lemma 3.1 only in a restricted range, and we need a different argument on the remaining part of the integration interval. This leads to some complications in details. In the conditional case, we have no such limitations and our result holds in the "natural" range for $H$. The details are in $\$ 5$, In both cases, we achieve the proof by removing the extraneous factor $\mathrm{e}^{-n / N}$ from the left-hand side of (8).

\section{LEMMAS}

It will shorten our formulae somewhat to write $\gamma_{k}=\Gamma(1+1 / k)$. For brevity, we also set

$$
\widetilde{\mathcal{E}}_{k}(\alpha):=\widetilde{S}_{k}(\alpha)-\frac{\gamma_{k}}{z^{1 / k}} \quad \text { and } \quad A(N ; c):=\exp \left\{c\left(\frac{\log N}{\log \log N}\right)^{1 / 3}\right\}
$$

where $c$ is a real constant.

Lemma 3.1 (Lemma 3 of [4], Lemma 1 of [2]). Let $\varepsilon$ be an arbitrarily small positive constant, $k \geq 1$ be an integer, $N$ be a sufficiently large integer and $L=\log N$. Then there exists a positive constant $c_{1}=c_{1}(\varepsilon)$, which does not depend on $k$, such that

$$
\int_{-\xi}^{\xi}\left|\widetilde{\mathcal{E}}_{k}(\alpha)\right|^{2} \mathrm{~d} \alpha \ll_{k} N^{2 / k-1} A\left(N ;-c_{1}\right)
$$

uniformly for $0 \leq \xi<N^{-1+5 /(6 k)-\varepsilon}$. Assuming the Riemann Hypothesis we have

$$
\int_{-\xi}^{\xi}\left|\widetilde{\mathcal{E}}_{k}(\alpha)\right|^{2} \mathrm{~d} \alpha \ll_{k} N^{1 / k} \xi L^{2}
$$

uniformly for $0 \leq \xi \leq 1 / 2$.

We remark that the proof of Lemma 3.1 in [4] contains oversights which are corrected in [6]. The next result is a variant of Lemma 4 of [4]: we just follow the proof until the last step. We need it to avoid dealing with the "periphery" of the major arc in the unconditional case. 
Lemma 3.2. Let $N$ be a positive integer, $z=z(\alpha)=1 / N-2 \pi \mathrm{i} \alpha$, and $\mu>0$. Then, uniformly for $n \geq 1$ and $X>0$ we have

$$
\int_{-X}^{X} z^{-\mu} \mathrm{e}(-n \alpha) \mathrm{d} \alpha=\mathrm{e}^{-n / N} \frac{n^{\mu-1}}{\Gamma(\mu)}+O_{\mu}\left(\frac{1}{n X^{\mu}}\right)
$$

Lemma 3.3. We have $\widetilde{S}_{k}(\alpha) \ll_{k} N^{1 / k}$.

Proof. It is a straightforward application of partial summation and a crude form of the Prime Number Theorem. In fact, recalling that the summatory function of the von Mangoldt $\Lambda$ function $\psi(X)$ satisfies $\psi(X) \ll X$, we have

$$
\begin{aligned}
\sum_{n \geq 1} \Lambda(n) \mathrm{e}^{-n^{k} / N} & =\lim _{X \rightarrow+\infty} \sum_{n \leq X} \Lambda(n) \mathrm{e}^{-n^{k} / N} \\
& =\lim _{X \rightarrow+\infty}\left(\psi(X) \mathrm{e}^{-X^{k} / N}+\frac{k}{N} \int_{0}^{X} \psi(t) t^{k-1} \mathrm{e}^{-t^{k} / N} \mathrm{~d} t\right) \\
& \ll{ }_{k} N^{-1} \int_{0}^{+\infty} t^{k} \mathrm{e}^{-t^{k} / N} \mathrm{~d} t \ll_{k} N^{1 / k} \int_{0}^{+\infty} u^{1 / k} \mathrm{e}^{-u} \mathrm{~d} u
\end{aligned}
$$

by a trivial change of variables.

Our next tool is Lemma 6 of Languasco \& Zaccagnini [5]: it is a consequence of Lemma 4 of [1], which depends, essentially, on a result of Robert \& Sargos [7].

Lemma 3.4. For $N$ a positive integer, $\tau>0$ and for real $k>1$ and real $\varepsilon>0$ we have

$$
\int_{-\tau}^{\tau}\left|\widetilde{S}_{k}(\alpha)\right|^{4} \mathrm{~d} \alpha \ll_{k}\left(\tau N^{2 / k}+N^{4 / k-1}\right) N^{\varepsilon}
$$

Lemma 3.5. For $k>1$ and $N^{-c}<\tau \leq N^{2 / k-1}$ we have

$$
\int_{\tau}^{1 / 2}\left|\widetilde{S}_{k}(\alpha)\right|^{4} \frac{\mathrm{d} \alpha}{\alpha} \ll_{k} N^{4 / k-1+\varepsilon} \tau^{-1}
$$

Proof. We just need a partial integration from Lemma 3.4; let

$$
F(\xi)=\int_{0}^{\xi}\left|\widetilde{S}_{k}(\alpha)\right|^{4} \mathrm{~d} \alpha \ll_{k}\left(\xi N^{2 / k}+N^{4 / k-1}\right) N^{\varepsilon}
$$

Now since $\tau \leq N^{2 / k-1}$.

$$
\int_{\tau}^{1 / 2}\left|\widetilde{S}_{k}(\alpha)\right|^{4} \frac{\mathrm{d} \alpha}{\alpha}=\left[\frac{F(\alpha)}{\alpha}\right]_{\tau}^{1 / 2}+\int_{\tau}^{1 / 2} \frac{F(\alpha)}{\alpha^{2}} \mathrm{~d} \alpha \ll_{k} N^{4 / k-1+\varepsilon} \tau^{-1},
$$

Lemma 3.6. For $N \rightarrow+\infty, H \in[1, N]$ and a real number $\lambda$ we have

$$
\sum_{n=N+1}^{N+H} \mathrm{e}^{-n / N} n^{\lambda}=\frac{1}{\mathrm{e}} H N^{\lambda}+O_{\lambda}\left(H^{2} N^{\lambda-1}\right) .
$$

Proof. Using the approximation $\mathrm{e}^{-n / N}=\mathrm{e}^{-1}+O\left(H N^{-1}\right)$ introduces an error $O_{\lambda}\left(H^{2} N^{\lambda-1}\right)$. If $\lambda=0$ there is nothing left to prove. If $\lambda<1$ with $\lambda \neq 0$ we have

$$
n^{\lambda}-N^{\lambda}=\lambda \int_{N}^{n} t^{\lambda-1} \mathrm{~d} t \ll \lambda(n-N) N^{\lambda-1} \leq H N^{\lambda-1}
$$

for all $n \in[N+1, N+H]$, and we see that

$$
\sum_{n=N+1}^{N+H} n^{\lambda}=H N^{\lambda}+O_{\lambda}\left(H^{2} N^{\lambda-1}\right) .
$$


If $\lambda \geq 1$ the argument above proves that $n^{\lambda}-N^{\lambda} \ll_{\lambda} H(N+H)^{\lambda-1} \ll_{\lambda} H N^{\lambda-1}$ since $H \leq N$, and the conclusion follows.

\section{Proof of Theorem 1.1}

We need to introduce another parameter $B=B(N)$, defined as

$$
B=N^{2 \varepsilon}
$$

Ideally, we would like to take $B=1$, but we are prevented from doing this by the estimate in $\$ 4.3$. We let $\mathcal{C}=\mathcal{C}(B, H)=[-1 / 2,-B / H] \cup[B / H, 1 / 2]$. Recalling (8) we write

$$
\begin{aligned}
\sum_{n=N+1}^{N+H} \mathrm{e}^{-n / N} R_{k}(n)=\gamma_{k}^{k+1} & \int_{-B / H}^{B / H} \frac{U(-\alpha, H)}{z^{(k+1) / k}} \mathrm{e}(-N \alpha) \mathrm{d} \alpha \\
& +\int_{-B / H}^{B / H}\left(\widetilde{S}_{k}(\alpha)^{k+1}-\frac{\gamma_{k}^{k+1}}{z^{(k+1) / k}}\right) U(-\alpha, H) \mathrm{e}(-N \alpha) \mathrm{d} \alpha \\
& \quad+\int_{C} \widetilde{S}_{k}(\alpha)^{k+1} U(-\alpha, H) \mathrm{e}(-N \alpha) \mathrm{d} \alpha \\
= & \gamma_{k}^{k+1} I_{1}+I_{2}+I_{3},
\end{aligned}
$$

say. The first summand gives rise to the main term via Lemma 3.2, the second one is majorised by means of identity (2) and the $L^{2}$-estimate provided by Lemma 3.1, and the last one is easy to bound using Lemma 3.5 .

4.1. Evaluation of $I_{1}$. It is a straightforward application of Lemma 3.2, here we exploit the flexibility of having variable endpoints instead of the full unit interval. We have

$$
I_{1}=\int_{-B / H}^{B / H} \frac{U(-\alpha, H)}{z^{(k+1) / k}} \mathrm{e}(-N \alpha) \mathrm{d} \alpha=\frac{1}{\gamma_{k}} \sum_{n=N+1}^{N+H} \mathrm{e}^{-n / N} n^{1 / k}+O_{k}\left(\frac{H}{N}\left(\frac{H}{B}\right)^{(k+1) / k}\right) .
$$

We evaluate the sum on the right-hand side of (10) by means of Lemma 3.6 with $\lambda=1 / k$. Summing up, we have

$$
I_{1}=\frac{1}{\mathrm{e} \gamma_{k}} H N^{1 / k}+O_{k}\left(H^{2} N^{1 / k-1}+\frac{H}{N}\left(\frac{H}{B}\right)^{(k+1) / k}\right) .
$$

We can neglect the second summand in the error term since $H \leq N$ and $B \geq 1$.

4.2. Bound for $I_{2}$. We let $x=x(\alpha)=\widetilde{S}_{k}(\alpha)$ and $y=y(\alpha)=\gamma_{k} z^{-1 / k}$ and use (2). We recall the bounds (6) and (7), and Lemma 3.3. Using Lemma 3.1 and the Cauchy-Schwarz inequality where appropriate, we have

$$
\begin{array}{rl}
I_{2} & \ll_{k} H \sum_{j=1}^{k} \int_{-B / H}^{B / H}|x-y|^{2} \cdot|x|^{k-j} \cdot|y|^{j-1} \mathrm{~d} \alpha+H \int_{-B / H}^{B / H}|x-y| \cdot|y|^{k} \mathrm{~d} \alpha \\
\ll_{k} H & \sum_{j=1}^{k} \max _{\alpha}|x|^{k-j} \cdot \max _{\alpha}|y|^{j-1} \int_{-B / H}^{B / H}|x-y|^{2} \mathrm{~d} \alpha \\
& +H\left(\int_{-B / H}^{B / H}|x-y|^{2} \mathrm{~d} \alpha \int_{-B / H}^{B / H}|y|^{2 k} \mathrm{~d} \alpha\right)^{1 / 2} \\
\ll_{k} & H \sum_{j=1}^{k} N^{(k-j) / k} \cdot N^{(j-1) / k} N^{2 / k-1} A\left(N ;-c_{1}\right)
\end{array}
$$




$$
\begin{aligned}
& +H N^{1 / k-1 / 2} A\left(N ;-\frac{1}{2} c_{1}\right)\left(\int_{-B / H}^{B / H} \frac{\mathrm{d} \alpha}{|z|^{2}}\right)^{1 / 2} \\
& \ll_{k} H N^{1 / k} A\left(N ;-\frac{1}{2} c_{1}\right),
\end{aligned}
$$

where $c_{1}=c_{1}(\varepsilon)>0$ is the constant provided by Lemma 3.1, which we can use if $B / H<$ $N^{-1+5 /(6 k)-\varepsilon}$. Recalling the choice in (9), we see that we can take

$$
H>N^{1-5 /(6 k)+3 \varepsilon} \text {. }
$$

4.3. Bound for $I_{3}$. For $k \geq 3$ we have

$$
\begin{aligned}
I_{3}=\int_{C} \widetilde{S}_{k}(\alpha)^{k+1} U(-\alpha, H) \mathrm{e}(-N \alpha) \mathrm{d} \alpha & \ll_{k} \max _{\alpha \in[-1 / 2,1 / 2]}\left|\widetilde{S}_{k}(\alpha)\right|^{k-3} \int_{C}\left|\widetilde{S}_{k}(\alpha)\right|^{4} \frac{\mathrm{d} \alpha}{\alpha} \\
& \ll_{k} N^{(k-3) / k} \cdot N^{4 / k-1+\varepsilon}(B / H)^{-1} \ll_{k} N^{1 / k+\varepsilon} H / B
\end{aligned}
$$

by Lemmas 3.3 and 3.5 . This is $\ll_{k} N^{1 / k} H A\left(N ;-c_{1} / 2\right)$, by our choice in (9). For $k=2$ we can use a slightly different argument, based on Lemma 5 of [6]. We omit the details.

4.4. Completion of the proof. For simplicity, from now on we assume that $H \leq N^{1-\varepsilon}$. Summing up from (11), (12) and (14), we proved that

$$
\sum_{n=N+1}^{N+H} \mathrm{e}^{-n / N} R_{k}(n)=\frac{\gamma_{k}^{k}}{\mathrm{e}} H N^{1 / k}+O_{k}\left(H N^{1 / k} A\left(N ;-\frac{1}{2} c_{1}\right)\right),
$$

provided that (13) holds, since the other error terms are smaller in our range for $H$. In order to achieve the proof, we have to remove the exponential factor on the left-hand side, exploiting the fact that, since $H$ is "small," it does not vary too much over the summation range. We use a sort of bootstrapping argument: since $\mathrm{e}^{-n / N} \in\left[\mathrm{e}^{-2}, \mathrm{e}^{-1}\right]$ for all $n \in[N+1, N+H]$, we can easily deduce from (15) that

$$
\mathrm{e}^{-2} \sum_{n=N+1}^{N+H} R_{k}(n) \leq \sum_{n=N+1}^{N+H} \mathrm{e}^{-n / N} R_{k}(n) \ll_{k} H N^{1 / k} .
$$

We can use this weak upper bound to majorise the error term arising from the development $e^{-x}=1+O(x)$ that we need in the left-hand side of (15). In fact, we have

$$
\begin{aligned}
\sum_{n=N+1}^{N+H} \mathrm{e}^{-n / N} R_{k}(n) & =\sum_{n=N+1}^{N+H}\left(\mathrm{e}^{-1}+O\left((n-N) N^{-1}\right)\right) R_{k}(n) \\
& =\mathrm{e}^{-1} \sum_{n=N+1}^{N+H} R_{k}(n)+O_{k}\left(H^{2} N^{1 / k-1}\right) .
\end{aligned}
$$

Finally, substituting back into (15), we obtain the required asymptotic formula for $H$ as in the statement of Theorem 1.1.

\section{Proof of Theorem 1.2}

The proof of the conditional version of our result is easier since Lemma 3.1 applies to the full unit interval, and this partially spares us the trouble of dealing with two different ranges. Recalling (8), we write

$$
\sum_{n=N+1}^{N+H} \mathrm{e}^{-n / N} R_{k}(n)=\gamma_{k}^{k+1} \int_{-1 / 2}^{1 / 2} \frac{U(-\alpha, H)}{z^{(k+1) / k}} \mathrm{e}(-N \alpha) \mathrm{d} \alpha
$$




$$
+\int_{-1 / 2}^{1 / 2}\left(\widetilde{S}_{k}(\alpha)^{k+1}-\frac{\gamma_{k}^{k+1}}{z^{(k+1) / k}}\right) U(-\alpha, H) \mathrm{e}(-N \alpha) \mathrm{d} \alpha
$$

The main term is evaluated as in $\$ 4$, whereas for the secondary term we have recourse again to (2). By Lemma 3.2 with $X=1 / 2$ and Lemma 3.6 with $\lambda=1 / k$, we find that

$$
\begin{aligned}
\int_{-1 / 2}^{1 / 2} \frac{U(-\alpha, H)}{z^{(k+1) / k}} \mathrm{e}(-N \alpha) \mathrm{d} \alpha & =\frac{1}{\gamma_{k}} \sum_{n=N+1}^{N+H} \mathrm{e}^{-n / N} n^{1 / k}+O_{k}\left(\frac{H}{N}\right) \\
& =\frac{1}{\mathrm{e} \gamma_{k}} H N^{1 / k}+O_{k}\left(H^{2} N^{1 / k-1}\right) .
\end{aligned}
$$

For the secondary term we argue as above, setting $x=x(\alpha)=\widetilde{S}_{k}(\alpha)$ and $y=y(\alpha)=\gamma_{k} z^{-1 / k}$ and using (2). First we deal with the range $[-1 / H, 1 / H]$ :

$$
\begin{aligned}
\int_{-1 / H}^{1 / H} & \left(\widetilde{S}_{k}(\alpha)^{k+1}-\frac{\gamma_{k}^{k+1}}{z^{(k+1) / k}}\right) U(-\alpha, H) \mathrm{e}(-N \alpha) \mathrm{d} \alpha \\
& \ll H \sum_{j=1}^{k} \int_{-1 / H}^{1 / H}|x-y|^{2} \cdot|x|^{k-j} \cdot|y|^{j-1} \mathrm{~d} \alpha+H \int_{-1 / H}^{1 / H}|x-y| \cdot|y|^{k} \mathrm{~d} \alpha \\
& \ll_{k} H \sum_{j=1}^{k} \max _{\alpha}|x|^{k-j} \cdot \max _{\alpha}|y|^{j-1} \int_{-1 / H}^{1 / H}|x-y|^{2} \mathrm{~d} \alpha \\
& \quad+H\left(\int_{-1 / H}^{1 / H}|x-y|^{2} \mathrm{~d} \alpha \int_{-1 / H}^{1 / H}|y|^{2 k} \mathrm{~d} \alpha\right)^{1 / 2} \\
& \ll_{k} H \sum_{j=1}^{k} N^{(k-j) / k} \cdot N^{(j-1) / k} N^{1 / k} H^{-1} L^{2}+H N^{1 /(2 k)} H^{-1 / 2} L\left(\int_{-1 / H}^{1 / H} \frac{\mathrm{d} \alpha}{|z|^{2}}\right)^{1 / 2} \\
& \ll_{k} N L^{2}+H^{1 / 2} N^{1 / 2+1 /(2 k)} L .
\end{aligned}
$$

In the remaining range we use a partial-integration argument, in order to exploit the full force of (7). By Lemma 3.1 we have

$$
F(\xi):=\int_{0}^{\xi}\left|\widetilde{\mathcal{E}}_{k}(\alpha)\right|^{2} \mathrm{~d} \alpha \ll_{k} N^{1 / k} \xi L^{2}
$$

Hence

$$
\int_{1 / H}^{1 / 2}\left|\widetilde{\mathcal{E}}_{k}(\alpha)\right|^{2} \frac{\mathrm{d} \alpha}{\alpha}=\left[\frac{F(\alpha)}{\alpha}\right]_{1 / H}^{1 / 2}+\int_{1 / H}^{1 / 2} \frac{F(\alpha)}{\alpha^{2}} \mathrm{~d} \alpha \ll_{k} N^{1 / k} L^{3} .
$$

Choosing $x$ and $y$ as above and using (16), we see that the contribution from the range $[1 / H, 1 / 2]$ is

$$
\begin{aligned}
\int_{1 / H}^{1 / 2} & \left(\widetilde{S}_{k}(\alpha)^{k+1}-\frac{\gamma_{k}^{k+1}}{z^{(k+1) / k}}\right) U(-\alpha, H) \mathrm{e}(-N \alpha) \mathrm{d} \alpha \\
& \ll_{k} \sum_{j=1}^{k} \max _{\alpha}|x|^{k-j} \cdot \max _{\alpha}|y|^{j-1} \int_{1 / H}^{1 / 2}|x-y|^{2} \frac{\mathrm{d} \alpha}{\alpha}+\left(\int_{1 / H}^{1 / 2}|x-y|^{2} \frac{\mathrm{d} \alpha}{\alpha} \int_{1 / H}^{1 / 2}|y|^{2 k} \frac{\mathrm{d} \alpha}{\alpha}\right)^{1 / 2} \\
& \ll_{k} \sum_{j=1}^{k} N^{(k-j) / k} \cdot N^{(j-1) / k} N^{1 / k} L^{3}+\left(N^{1 / k} L^{3}\right)^{1 / 2} H \\
& \ll_{k} N L^{3}+H N^{1 /(2 k)} L^{3 / 2} .
\end{aligned}
$$


5.1. Completion of the proof. Summing up, we proved that

$$
\sum_{n=N+1}^{N+H} \mathrm{e}^{-n / N} R_{k}(n)=\frac{\gamma_{k}^{k}}{\mathrm{e}} H N^{1 / k}+O_{k}\left(\Phi_{k}(N, H)\right),
$$

where $\Phi_{k}(N, H)=N L^{3}+H N^{1 /(2 k)} L^{3 / 2}+H^{2} N^{1 / k-1}+H^{1 / 2} N^{1 / 2+1 /(2 k)} L$. As in $\$ 4.4$ above, we need to remove the exponential factor, exploiting the fact that, since $H$ is "small," it does not vary too much over the summation range. We argue in a slightly different fashion, since we aim at a stronger error term. Using the fact that $\mathrm{e}^{-n / N}=\mathrm{e}^{-1}+O(H / N)$, we have

$$
\sum_{n=N+1}^{N+H} R_{k}(n)=\gamma_{k}^{k} H N^{1 / k}+O_{k}\left(\Phi_{k}(N, H)+\frac{H}{N} \sum_{n=N+1}^{N+H} R_{k}(n)\right) .
$$

The last term is $\ll_{k} H / N\left(H N^{1 / k}+\Phi_{k}(N, H)\right) \ll_{k} H^{2} N^{1 / k-1}+\Phi_{k}(N, H) \ll_{k} \Phi_{k}(N, H)$ by (17), since $H \leq N$. Substituting into (18) we find

$$
\sum_{n=N+1}^{N+H} R_{k}(n)=\gamma_{k}^{k} H N^{1 / k}+O_{k}\left(\Phi_{k}(N, H)\right) .
$$

This is an asymptotic formula provided that $H=\infty\left(N^{1-1 / k} L^{3}\right)$ and $H=o(N)$. Theorem 1.2 is fully proved.

\section{Proof of Theorems 1.3 and 1.4}

We split the unit interval as in $\$ 4$ and proceed in the same way. With a similar notation, we find that

say, where

$$
\sum_{n=N+1}^{N+H} \mathrm{e}^{-n / N} R_{k}^{\prime}(n)=\gamma_{k}^{k} I_{1}^{\prime}+I_{2}^{\prime}+I_{3}^{\prime}
$$

$$
I_{1}^{\prime}=H \mathrm{e}^{-1}+O_{k}\left(H^{2} / N+H / B\right),
$$

by Lemma 3.2 and standard estimates. We also have

$$
I_{2}^{\prime} \ll_{k} H A\left(N ;-\frac{1}{2} c_{1}\right),
$$

arguing as above by means of identity (2) with $k$ replaced by $k-1$. For $k \geq 4$ we have

$$
I_{3}^{\prime}=\int_{C} \widetilde{S}_{k}(\alpha)^{k} U(-\alpha, H) \mathrm{e}(-N \alpha) \mathrm{d} \alpha \ll_{k} \max _{\alpha \in[-1 / 2,1 / 2]}\left|\widetilde{S}_{k}(\alpha)\right|^{k-4} \int_{C}\left|\widetilde{S}_{k}(\alpha)\right|^{4} \frac{\mathrm{d} \alpha}{\alpha} \ll_{k} N^{\varepsilon} H / B,
$$

by Lemmas 3.3 and 3.5, which is $\ll_{k} H A\left(N ;-c_{1} / 2\right)$, by our choice of $B$.

For $k \in\{2,3\}$ we need the extension to $\widetilde{S}_{3}$ of Lemma 7 of Tolev [8]. The details of the proof are contained in work in progress by A. Languasco and the last Author. Without this Lemma, for $k=3$ we need to take $B=N^{5 / 72+\varepsilon}$ and we obtain a correspondingly weaker result.

Lemma 6.1 (Tolev). Let $k>1$ and $0<\tau \leq 1 / 2$. Then

$$
\int_{-\tau}^{\tau}\left|\widetilde{S}_{k}(\alpha)\right|^{2} \mathrm{~d} \alpha \ll_{k}\left(\tau N^{1 / k}+N^{2 / k-1}\right)(\log N)^{3} .
$$

A partial-integration argument similar to the ones above then yields

$$
I_{3}^{\prime} \ll_{k} \max _{\alpha \in[-1 / 2,1 / 2]}\left|\widetilde{S}_{3}(\alpha)\right|^{k-2} \int_{C}\left|\widetilde{S}_{k}(\alpha)\right|^{2} \frac{\mathrm{d} \alpha}{\alpha} \ll_{k} N^{(k-2) / k}\left(N^{1 / k} L^{4}+\frac{H}{B} N^{(2-k) / k} L^{3}\right),
$$

which is $o\left(H N^{-\varepsilon}\right)$ if $B=N^{2 \varepsilon}$ as in (9), for $H$ as in the statement of Theorem 1.3 ,

We omit the details of the proof of Theorem 1.4 . 
Acknowledgement. We thank Alessandro Languasco for several conversations on this topic.

\section{REFERENCES}

[1] A. Gambini, A. Languasco, and A. Zaccagnini, A Diophantine approximation problem with two primes and one $k$-th power of a prime, J. Number Theory 188 (2018), 210-228.

[2] A. Languasco and A. Zaccagnini, A Diophantine problem with prime variables, Highly Composite: Papers in Number Theory, Proceedings of the "International Meeting in Number Theory," celebrating the 60th birthday of Prof. R. Balasubramanian, Harish-Chandra Research Institute, Allahabad, Dec. 2011 (V. Kumar Murty, D. S. Ramana, and R. Thangadurai, eds.), Ramanujan Mathematical Society-Lecture Notes Series, vol. 23, 2016, pp. $157-168$.

[3] A. Languasco and A. Zaccagnini, Short intervals asymptotic formulae for binary problems with primes and powers, II: density 1, Monatsh. Math. 181 (2016), no. 3, 419-435.

[4] A. Languasco and A. Zaccagnini, Sum of one prime and two squares of primes in short intervals, J. Number Theory 159 (2016), 45-58.

[5] A. Languasco and A. Zaccagnini, Sums of four prime cubes in short intervals, Acta Math. Hung. (2019). Accepted for publication. Arxiv preprinthttp://arxiv.org/abs/1705.04457.

[6] A. Languasco and A. Zaccagnini, Sums of one prime power and two squares of primes in short intervals, Rocky Mountain J. Math. (2019). Accepted for publication. Arxiv preprint http://arxiv.org/abs/1806.04934,

[7] O. Robert and P. Sargos, Three-dimensional exponential sums with monomials, J. reine angew. Math. 591 (2006), 1-20.

[8] D. I. Tolev, On a Diophantine inequality involving prime numbers, Acta Arith. 51 (1992), 289-306.

Dipartimento di Scienze, Matematiche, Fisiche e Informatiche

Università di Parma

Parco Area delle Scienze 53/a

43124 Parma, Italia

email (MC): cantarini_melibero.it

email (AG): a.gambini@unibo.it

email (AZ): alessandro.zaccagnini@unipr.it 